\title{
A systematic review of pediatric clinical trials of high dose vitamin D
}

Nassr Nama, Kusum Menon, Klevis Iliriani, Supichaya Pojsupap, Margaret Sampson, Katie O'Hearn, Linghong (Linda) Zhou, Lauralyn McIntyre, Dean Fergusson, James D McNally

Background. Due to inadequate UV exposure, intake of small quantities of vitamin D is recommended to prevent musculoskeletal disease. Both basic science and observational literature strongly suggest that higher doses may benefit specific populations and have non-musculoskeletal roles. Evaluating the evidence surrounding high dose supplementation can be challenging given a relatively large and growing body of clinical trial evidence spanning time, geography, populations and dosing regimens. Study objectives were to identify and summarize the clinical trial literature, recognize areas with high quality evidence, and develop a resource database that makes the literature more immediately accessible to end users.

Methods. Medline (1946 to January 2015), Embase (1974 to January 2015), and Cochrane databases (January 2015), were searched for trials. All pediatric (0-18 years) trials administering doses higher than $400 \mathrm{IU}$ ( $<1$ year) or $600 \mathrm{IU}$ ( $\geq 1$ year) were included. Data was extracted independently by two of the authors. An online searchable database of trials was developed containing relevant extracted information ( https://vitamind.knackhq.com/pediatrics ). Sensitivity and utility were assessed by comparing the trials in the database to those from systematic reviews of vitamin $D$ supplementation including children.

Results. 2579 candidate papers were identified, yielding 169 trials having one or more arms meeting eligibility criteria. The publication rate has increased significantly from 1 per year (1970-1979) to 14 per year (2010-2015). Although $84 \%$ of the total trials focused on healthy children or known high risk populations (e.g. renal, prematurity), this proportion has declined in recent years due to the rise in trials evaluating populations and outcomes not directly related to the musculoskeletal actions of vitamin D ( $27 \%$ in 2010 s). Beyond healthy children, the only pediatric populations with more than 50 participants from low risk of bias trials evaluating a clinically relevant outcome were prematurity and respiratory illness. Finally, we created and validated the online searchable database using 13 recent systematic reviews. Of the 38 high dose trials identified by the systematic review, 36 $(94.7 \%)$ could be found within the database. When compared with the search strategy reported in each systematic review, use of the database reduced the number of full papers 
to assess for eligibility by $85.2 \%( \pm 13.4 \%)$.

Conclusion. The pediatric vitamin D field is highly active, with a significant increase in trials evaluating non-classical diseases and outcomes. Despite the large overall number there are few high quality trials of sufficient size to provide answers on clinical efficacy of high-dose vitamin D. An open access online searchable data should assist end users in the rapid and comprehensive identification and evaluation of trials relevant to their population or question of interest. 


\section{A systematic review of pediatric clinical trials of high dose vitamin D}

2

3 Nassr Nama ${ }^{1,2}$, Kusum Menon ${ }^{2}$, Klevis Iliriani ${ }^{3}$, Supichaya Pojsupap ${ }^{2}$, Margaret Sampson ${ }^{4}$, Katie

$4 \mathrm{O}^{\prime} \mathrm{Hearn}^{2}$, Linghong Zhou ${ }^{1}$, Lauralyn McIntyre ${ }^{4}$, Dean Fergusson ${ }^{4}$, and James Dayre McNally ${ }^{1,2}$

$7 \quad{ }^{1}$ Faculty of Medicine, University of Ottawa, Ottawa, Ontario, Canada

$8 \quad{ }^{2}$ Department of Pediatrics, Children's Hospital of Eastern Ontario, Ottawa, Ontario, Canada

$9 \quad{ }^{3}$ School of Medicine, Trinity College, Dublin, Ireland

$10{ }^{4}$ Department of Volunteers, Communication and Information Resources, Children's Hospital of

11 Eastern Ontario, Ottawa, Ontario, Canada

$12{ }^{5}$ Ottawa Hospital Research Institute, Ottawa, Ontario, Canada

13

14 Corresponding author:

15 James Dayre McNally ${ }^{1,2}$

16401 Smyth Road, Ottawa, Ontario, Canada, K1H 8L1

17 E-mail: dmcnally@cheo.on.ca 


\section{Abstract}

20 Background. Due to inadequate UV exposure, intake of small quantities of vitamin D is

21 recommended to prevent musculoskeletal disease. Both basic science and observational literature

22 strongly suggest that higher doses may benefit specific populations and have non-

23 musculoskeletal roles. Evaluating the evidence surrounding high dose supplementation can be

24 challenging given a relatively large and growing body of clinical trial evidence spanning time,

25 geography, populations and dosing regimens. Study objectives were to identify and summarize

26 the clinical trial literature, recognize areas with high quality evidence, and develop a resource

27 database that makes the literature more immediately accessible to end users.

28 Methods. Medline (1946 to January 2015), Embase (1974 to January 2015), and Cochrane

29 databases (January 2015), were searched for trials. All pediatric (0-18 years) trials administering

30 doses higher than $400 \mathrm{IU}(<1$ year) or $600 \mathrm{IU}(\geq 1$ year) were included. Data was extracted

31 independently by two of the authors. An online searchable database of trials was developed

32 containing relevant extracted information (https://vitamind.knackhq.com/pediatrics). Sensitivity

33 and utility were assessed by comparing the trials in the database to those from systematic

34 reviews of vitamin D supplementation including children.

35 Results. 2579 candidate papers were identified, yielding 169 trials having one or more arms

36 meeting eligibility criteria. The publication rate has increased significantly from 1 per year

37 (1970-1979) to 14 per year (2010-2015). Although $84 \%$ of the total trials focused on healthy

38 children or known high risk populations (e.g. renal, prematurity), this proportion has declined in

39 recent years due to the rise in trials evaluating populations and outcomes not directly related to

40 the musculoskeletal actions of vitamin D (27\% in 2010s). Beyond healthy children, the only

41 pediatric populations with more than 50 participants from low risk of bias trials evaluating a 
42 clinically relevant outcome were prematurity and respiratory illness. Finally, we created and

43 validated the online searchable database using 13 recent systematic reviews. Of the 38 high dose

44 trials identified by the systematic review, 36 (94.7\%) could be found within the database. When

45 compared with the search strategy reported in each systematic review, use of the database

46 reduced the number of full papers to assess for eligibility by $85.2 \%( \pm 13.4 \%)$.

47 Conclusion. The pediatric vitamin D field is highly active, with a significant increase in trials

48 evaluating non-classical diseases and outcomes. Despite the large overall number there are few

49 high quality trials of sufficient size to provide answers on clinical efficacy of high-dose vitamin

50 D. An open access online searchable data should assist end users in the rapid and comprehensive

51 identification and evaluation of trials relevant to their population or question of interest.

\section{Introduction}

Vitamin D is a steroid-based hormone familiar to health care providers, patients and the media. It is well established that appropriate body stores of vitamin D are essential to musculoskeletal health (Parfitt et al., 1982; Beck-Nielsen et al., 2009; Melamed \& Kumar, 2010). As reliance solely on sun (UV) exposure results in high rates of vitamin D deficiency (Robinson et al., 2006; Ahmed et al., 2011; Merewood et al., 2012; Thacher, Fischer \& Pettifor, 2014), multiple scientific agencies have recommended daily supplementation with small quantities of vitamin D (Ross et al., 2011). Despite the success of this approach in reducing the incidence of vitamin D deficiency related electrolyte disturbances and rickets, there continues to be significant interest in alternative high dose vitamin D supplementation strategies. Potential explanations include concern that specific pediatric populations remain at risk for vitamin D deficiency despite recommended dosing (Aguirre Castaneda et al., 2012) and that higher doses of vitamin D may protect against or improve outcomes for a wide range of non-musculoskeletal 
65 diseases involving the immune, respiratory, and cardiovascular systems (Brehm et al., 2010;

66 Levin et al., 2011; Gray et al., 2012; Abrams, Coss-Bu \& Tiosano, 2013; McNally et al., 2015;

67 Tomaino et al., 2015; Cadario et al., 2015). Presumably due to uncertainty surrounding the

68 benefit of and best approach to vitamin D supplementation, there has been a growing body of

69 pediatric clinical trial literature. This work spans time, geography, populations (disease states),

70 dosing regimens, and outcome measures. These factors, combined with the large number of adult

71 trials, animal studies and observational literature make the available evidence difficult to find,

72 synthesize and translate to clinical practice or cutting edge research. To assist clinicians and

73 researchers we have sought to identify, describe, and quantify the existing clinical trial literature

74 of high-dose vitamin D supplementation in children through the completion of a systematic

75 review.

76 The objective of this systematic review was to describe the populations, dosing regimens,

77 methodologies and outcome measures and evaluate how they have varied across geography and

78 time. In addition, we sought to determine the areas where there may be sufficient quantity of

79 high quality evidence to evaluate the benefits of high-dose vitamin D on clinically relevant

80 outcomes. Finally, to assist end user groups in the identification of trials relevant to their specific

81 patient populations, policy development or research areas, we sought to develop an online trial

82 database searchable by keyword and study characteristics.

\section{Material and Methods}

84 Study protocol and objectives were established a priori (PROSPERO protocol registration

85 number: CRD42015016242) and reported here according to the PRISMA guidelines of

86 systematic reviews (Table S1) (Moher et al., 2009). 


\section{Eligibility Criteria}

Studies were eligible for inclusion in this systematic review if they satisfied all of the following criteria: (1) Uncontrolled, controlled non-randomized, or randomized controlled trial (RCT); (2) the study involved children; and (3) the study administered cholecalciferol (D3) or ergocalciferol (D2) above the Institute of Medicine (IOM) age specific Recommended Dietary Allowance (RDA) or Adequate Intake (AI). The AI (infants) has been set at 400IU, with RDA set at $600 \mathrm{IU}$ for those older than one year. As adequate dosing in low birth weight and premature neonates is less well defined, trials administering any dose were considered eligible in these populations. Only trials published in English, French, Spanish, or German were included. Studies were excluded if: they administered Vitamin D as part of a formula, or mixed with food, and dosage was not controlled or consistently delivered; there were no patients less than or equal to 15 years of age; or the study group included patients older than 18 years and did not present data separately for children and adults.

\section{Identification of Studies}

The search strategy has been previously described (McNally et al., 2015). Medline (1946), Embase (1970), and the Cochrane Central Register of Controlled Trials (2005) were searched in January 2014 and updated in January 2015 using the Ovid interface. No date, language, or study design limits were applied to the electronic search. The grey literature search included a citation review of all eligible articles, and 24 systematic reviews of vitamin D in children (Appendix S1).

The Medline search strategy (Appendix S2) was developed by a librarian (MS) and peer reviewed by another (Lorie Kloda, MLIS, PhD), using the PRESS (Peer Review of Electronic Search Strategies) standard (Sampson et al., 2009).

Two of the study authors independently reviewed the citations through three sets of 
111 screening questions to determine eligibility (Table S2). Level 1 screening was performed using

112 Mendeley (Mendeley Desktop, version 1.13.8), and those citations that could not be excluded

113 were uploaded to DistillerSR (Evidence Partners, Inc., Ottawa, Canada) for the second and third

114 levels of screening where the full text was assessed for eligibility by two authors, with conflicts

115 resolved by a third. A single author determined the eligibility of articles written in German or

116 Spanish. In the situation of a trial having produced multiple publications, we selected the largest

117 or most complete report; if the two reports described different outcomes in the same trial, all

118 assessed outcomes where listed under the largest report.

\section{Data Collection and Analysis}

120 Data was extracted from eligible articles and reviewed independently by two of the authors

121 (N.N., K.I., K.O., S.P., J.D.M.). Data was collected using the REDCap system (Research

122 Electronic Data Capture) (Harris et al., 2009). Any 25-hydroxyvitamin D (25OHD) data reported

123 only in graphs was extracted using DigitizeIt software (http://www.digitizeit.de/, Germany).

124 Study populations were stratified into three groups: i) Healthy children with the level of 25OHD

125 and/or bone health as a primary outcome, ii) diseases classically linked to vitamin D or known to

126 affect its pharmacokinetics (prematurity, renal, rickets, malabsorption, epileptic medications)

127 (Canadian Agency for Drugs and Technologies in Health, 2015), and iii) studies involving

128 children with non-classical diseases or targeting non-classical outcomes. This stratification of

129 classical vs. non-classical diseases follows the guidelines established by several endocrine

130 societies. This separation is relevant as testing for 25OHD levels may only be funded (Canada

131 for example) in patients with one of the conditions classically associated with vitamin D

132 deficiency (Provincial Programs Branch, Goverment of Ontario, 2010; Canadian Agency for

133 Drugs and Technologies in Health, 2015). 
134 Vitamin D dosing regimens were placed into one of three frequency groups (daily,

135 weekly/bi-weekly, and single/intermittent) and 4 dosing groups (< 1000 IU, 1000-3999, 4000-

$13639999,>40000 \mathrm{IU})$ categories. Consistent with our systematic review evaluating change in

$13725 \mathrm{OHD}$ by dosing regimen, results were presented by study arm, as publications frequently

138 evaluated more than one high dose regimen (McNally et al., 2015). Where applicable, we also

139 identified whether and how trials varied the dose (e.g. weight, age or body surface area (BSA))

140 and determined the maximum dose administered based on the description provided for the study

141 participants in the results. Each study was assessed using Cochrane risk of bias tool (Higgins \&

142 Green, 2011). Areas where there might be sufficient high quality research to address clinical

143 efficacy were determined by cross-referencing low risk of bias studies with population-outcome

144 data and number of participants enrolled.

145 Statistical Analysis

146 Data analysis was performed using SAS (version 9.3; SAS Institute, Cary, NC) and

147 GraphPad Prism (version 6.0.5; GraphPad Software, Inc. La Jolla, CA). Figures were generated

148 using SigmaPlot (version 12.3.0.36; Systat Software, Inc. Germany). Chi-square and Fisher's

149 exact tests were used to compare features between different decades and/or regions.

150 Online Database

151 Using Knack software, we developed an online database with relevant information

152 extracted from each identified trial (https://vitamind.knackhq.com/pediatrics). Twenty-one

153 systematic reviews reporting on vitamin D supplementation in children from 2008 to 2013 were

154 evaluated and all population, dosing, outcome and methodology characteristics reported in more

155 than $1 / 3$ of these systematic reviews were included in the online database (Dataset S1). 
156 Validity, Utility and Accessibility

157 Comprehensiveness of the database was evaluated using the search results from 13

158 systematic reviews (Table 5) not included in the original literature search (McNally et al., 2015).

159 To be included in the validation, the systematic review had to: (1) assess trials of vitamin D

160 supplementation; and (2) contain at least one prospective pediatric trial. Systematic reviews were

161 excluded if they were published by one of our authors, or the reference list was screened as part

162 of the literature search for our previous systematic review (McNally et al., 2015). Validation was

163 performed by an independent author (LZ), who was not involved in the development of the

164 database, and was blinded to the search results of the individual systematic reviews. This

165 individual was provided with the eligibility criteria for each systematic review and then used the

166 online database to identify the list of trials that would need further screening. Trial eligibility was

167 further confirmed by a second author (NN). Sensitivity of the database was determined by

168 comparing the number of trials in the database to the number identified within the individual

169 systematic reviews (gold standard). Utility of the database was assessed by determining whether

170 application of the database would have reduced the number of abstracts and full text articles for

171 review. This was performed by a blinded author and using the population, age and outcome

172 filters. Finally, accessibility was assessed using the Minervation validation instrument for

173 healthcare websites (LIDA tool). This validation tool has been previously validated and used in

174 several fields (Nankervis, Maplethorpe \& Williams, 2011; Pithon \& Santos, 2014; Carlsson et

175 al., 2015; Redmond et al., 2015; Küçükdurmaz et al., 2015). Accessibility is addressed by

176 looking at page setup, access restrictions, amount of outdated code and compatibility with NHS

177 directives.

178 Results 


\section{Search Results}

180 Figure 1 demonstrates the flow of studies identified by the search strategy. In total, 2304

181 unique records were retrieved from the original electronic search with an additional 146 citations

182 found in the reference lists of systematic reviews and eligible articles. Updating the search in

183 January 2015 added an additional 129 records. Of the 2579 articles, 2188 were excluded at level

184 one, with an additional 135 excluded at level two screening. In total, we identified 256

185 publications that reported on the results of a clinical trial administering any dose of

186 ergocalciferol or cholecalciferol to children. From these 256 articles, 169 articles met eligibility

187 criteria (Appendix S3). Further inspection identified 6 instances of double publications of the

188 same trial with reporting of nearly identical findings (Table S3). These duplicate publications

189 were not included in the study, resulting in a total of 163 publications.

190 Study Design, Participant Number and Quality

191 The 163 publications evaluated 181 distinct study populations, included 365 separate arms, 192 and enrolled a total of 18539 children (Fig. S1). As shown in Table 1, RCTs contributed to the

193 majority of the trials $(n=108 / 163,66 \%)$ and patients $(n=15728,84.8 \%)$. Assessment of trial

194 quality determined that $23 \%(\mathrm{n}=38 / 163)$ and $42 \%(\mathrm{n}=69 / 163)$ were at low or medium/uncertain

195 risk for bias, respectively. Of the 365 study arms, 263 (72.1\%) administered one or more doses

196 of vitamin D meeting our eligibility criteria, on a total of 11947 children. The median number of

197 participants in the high dose arms was 25 (IQR: 14 - 42). The 163 trials were published over a

198 46-year period between 1969 and 2014 (inclusive). The rate of trial publication changed

199 significantly over time $(\mathrm{p}<0.001)$, increasing from 1 trial per decade (1960-1969) to 15 trials in

2002014 alone (Fig. 2A). Of the 163 trials, almost half $(n=72,44 \%)$ have been published in the last

2015 years (2010 to 2014). Compared to a linear model, the change over time better fits an 
202 exponential function with the number of trials doubling every 12.7 years $\left(\mathrm{R}^{2}=0.85\right.$ vs. 0.96 203 respectively).

204 Populations

205 The number and percentage of study arms recruiting populations within five age categories

206 is provided in Table 1. Specific age categories were targeted in 61 arms, with the majority of

207 those focusing on neonates $(n=47 / 61,77.0 \%)$. Study arms involving populations with classic 208 vitamin D related diseases were the largest category $(n=123 / 263,46.8 \%)$, with prematurity $209(\mathrm{n}=48,18.3 \%)$ and rickets $(\mathrm{n}=43,16.3 \%)$ representing the most common subpopulation (Table

210 2). Of the remaining 140 high-dose arms, 97 recruited healthy patients and focused on a classical

211 role of vitamin D (25OHD or bone health) and/or prevention of rickets. The least common

212 category, representing $16.3 \%$ of arms $(n=43 / 263)$ were those with diseases or outcomes less

213 classically related to vitamin D. Of these, 12 included healthy patients and focused on primary

214 prevention of non-classical conditions (respiratory infections, diabetes), and the remaining 31

215 enrolled participants with wide range of non-classical illness at baseline (Table S4), and

216 administered vitamin D as a sole or part of the treatment plan. The proportion of arms evaluating

217 non-classical populations or outcomes has increased significantly ( $p<0.001$ ), rising to $26.7 \%$

218 during the current decade (Fig. 2B). The number of low risk of bias arms enrolling more than 50

219 participants is presented in Table 2.

220

221 Dosing Regimens

222 Evaluation of dosing regimen characteristics identified the main supplement and route as

223 cholecalciferol $(n=162 / 263,61.6 \%)$ and enteral $(n=238 / 263,90.5 \%)$, respectively (Table 3 ).

224 Regarding supplementation frequency, 137 arms (52.1\%) delivered drug on a daily schedule, and 
$22596(36.5 \%)$ used intermittent loading therapy. Most of the arms used a constant dose of vitamin

$226 \mathrm{D}(\mathrm{n}=224 / 263,85.2 \%)$, while the remaining used dosing based on age/weight or body surface

227 area $(n=27 / 263,10.3 \%)$, baseline $250 H D(n=7 / 263,2.7 \%)$, or initial response to

228 supplementation ( $\mathrm{n}=5 / 263,1.9 \%$ ). Doses higher than 40,000 IU were the most common dose

229 group, being used in 107 arms (40.7\%). Whether dosing regimen characteristics changed over

230 time was further investigated (Figure 2C); ergocalciferol (D2) was more common prior to the

231 1980s, with cholecalciferol (D3) gaining significant attention over the past two decades

$232(\mathrm{p}<0.001)$. In the present decade, D3 and D2 were used in $(n=90 / 105,86 \%)$ and $(n=10 / 105$,

$23310 \%$ ) of the high-dose arms respectively (remainder unclear). No other change over time was

234 evident for frequency of administration, choice of variable dosing, or route of administration

235 (Fig. S2).

236 Geographical Regions

237 Trials were categorized by geographical region (Fig. 3A) $(\mathrm{p}<0.001)$. The area with the 238 most published trials was Europe (n=46/163, 28\%) with North America and the Middle East

239 each contributing 35 trials (21\%). For comparison, the number of total participants by

240 geographical region is shown in Fig. 3B $(\mathrm{p}<0.001)$. Difference in dosing regimen preference by

241 geographical region was also evident. Doses higher than 40,000 IU were sparse in Europe

$242(n=14 / 263,5.3 \%)$ and North America $(n=9 / 263,3.4 \%)$, but represented the most commonly used

243 regimen in every other region (Fig. S3). In addition to dose, statistically significant differences

244 were observed between North America and Europe when compared to the rest of the world for

245 route, form and dosing frequency (Fig. 3). A comparison of the population types studied in these

246 two continents, with the remainder of the world, did not identify statistically significant

247 differences $(\mathrm{p}=0.81)$ (Fig. 3G). 


\section{Outcomes}

249 Primary outcome varied among trials, with $106(65 \%)$ targeting a biochemical marker, and

$25062(38 \%)$ focusing on a clinical outcome (Table 4). Considering all outcomes reported, 25OHD

251 level was the most common, being studied in 133 trials $(82 \%)$, with blood calcium $(\mathrm{n}=118 / 163$,

$25272 \%)$, phosphate $(n=80 / 163,49 \%)$ and PTH $(n=69 / 163,42 \%)$ being the next most common. For

253 studies focused on non-classical diseases, outcomes evaluating the immunologic, respiratory and 254 cardiovascular systems were studied in $19(12 \%), 9(6 \%)$ and $8(5 \%)$ trials.

\section{Database validation}

The thirteen systematic reviews identified 38 trials meeting our high-dose criteria, 36

257

258

259

260

261

262

263

264

265

266

267

268

269

270

$(94.7 \%)$ of which were contained within the online searchable database. The database identified an additional 16 trials that satisfied the inclusion criteria of one or more of these systematic reviews, and were published prior to the literature search. The two eligible trials not present within the database were determined to be not available as a full text (abstract) or the article could not be located $(n=1$, the reference did not exist on any of the searched databases or the journal repertoire). The 13 systematic reviews also included an additional 18 trials that provided supplementation with lower doses of vitamin D $(\leq$ RDA $)$, and all were present in the list of 256 pediatric clinical trials identified as part of the stage 2 screening. A summary of the utility assessment is shown in Table S5. The literature search of the 4 full pediatric systematic reviews identified between 684 and 1343 unique records for screening and between 21 and 274 articles for full text review. In comparison, the online database search by the blinded author and using the population, age and outcome filters yielded between 2 and 10 articles for full text review (no eligible studies were missed). The reduction in number of papers for full assessment was reduced by $85.2 \%(\mathrm{SD} 13.4 \%)$. 

setup, access restrictions, amount of outdated code and compatibility. On this, our online

273 database scored 45/54 (83\%) in terms of accessibility (Table S6).

\section{Discussion} vitamin D supplementation, and determined this to be a large and rapidly expanding area of

277 clinical research. Descriptive analysis identified heterogeneity in important trial design characteristics, including a recent significant increase in studies evaluating a wide range of populations and outcomes not classically related to vitamin D. A comprehensive searchable online database was developed to aid clinicians and researchers in the identification and evaluation of trials relevant to their patient population or area of interest. of which 169 included one or more study arms meeting our definition of high dose. Evaluation of publications over time further demonstrated high-dose vitamin D supplementation to be a rapidly expanding area of clinical research. With the exception of the 1990's, where a brief decline in publications was observed, the trial number has almost doubled each decade. The decline in publications on high-dose vitamin D may relate to a late 1980's publication reporting high hypercalcemia rates (34\%) in young infants receiving 600000 IU (Markestad et al., 1987). was limited by the lack of well-done systematic reviews of similar design and scope. However, one excellent comparator is the recent review published by Duffett and colleagues, wherein they

292 demonstrated a constant linear rise in clinical trials in the pediatric critical care setting (Duffett et

293 al., 2013). Taken together, the results suggest that pediatric clinical trial literature is steadily 
294 increasing. The exponential growth of the randomized controlled trial literature has been

295 previously demonstrated (Tsay \& Yang, 2005; Bastian, Glasziou \& Chalmers, 2010). Part of the

296 value of systematic reviews is in summarizing the literature, as the rate of publication of RCTs

297 makes it impractical for clinicians to keep up with the primary publications.

298 Further comparison of publication rates between these two pediatric studies suggested a

299 faster rate of rise in trials on high dose vitamin D. For perspective, if the current rate is

300 maintained there will have been more trials published between 2010 and 2019 then in the

301 preceding 5 decades combined. Evaluation of study characteristics, including the change over

302 time, provided some insight into why the publication rate may be rising faster than other

303 areas.Importantly, this evaluation demonstrated that an increasing number of trials are focusing

304 on populations or outcomes not classically related to vitamin D (Canadian Agency for Drugs and

305 Technologies in Health, 2015). This shift is consistent with the substantial growth in

306 observational literature over the past two decades linking vitamin $\mathrm{D}$ to a widespread number of

307 disorders involving the immune, neurological, respiratory, and cardiovascular systems (Brehm et

308 al., 2010; Levin et al., 2011; Gray et al., 2012; McNally et al., 2012; Abrams, Coss-Bu \&

309 Tiosano, 2013; Cadario et al., 2015). The decision to pursue clinical trials of high dose

310 supplementation in these populations may relate to postulations made that higher 25OHD levels,

311 relative to those achieved with RDA, may be required to achieve maximal benefit for non-

312 musculoskeletal outcomes (Hathcock et al., 2007). Further, available literature also suggests that

313 when compared to healthy patients, those with acute and chronic disease may have a blunted

314 response to usual doses of vitamin D (McNally et al., 2015).

315 As part of the descriptive analysis we also sought out heterogeneity in other trial design

316 features, including dosing regimen characteristics. The main dosing regimen characteristics 
317 where uniformity was evident include use of the oral route for drug administration and choice of

318 the cholecalciferol form. Consistency in these areas is expected, given that cholecalciferol has

319 been suggested to have favorable metabolism and greater biological activity, and 25OHD is well

320 accepted as the best biological marker of vitamin D status (Melamed \& Kumar, 2010). Outside

321 of route and form, significant heterogeneity in regimen dose and frequency was evident. Further

322 exploration determined that some of the heterogeneity could be explained by the geographical

323 origin of the trial. In North America and Europe, daily supplementation was by far the most

324 common regimen, while the remainder of the world predominantly used single or divided

325 loading doses well in excess of the IOM Daily Upper Tolerable Intake Level. These differences

326 might explain why, in contrast to countries like Australia and New Zealand, guidelines

327 originating out of North America do not offer any strategies based on weekly or less frequent

328 loading doses (Munns et al., 2006; Godel, 2007; Manaseki-Holland et al., 2010). Similar to the

329 shift from ergocalciferol to cholecalciferol over time, a study characteristic anticipated to be

330 changing was the proportion of dosing regimens incorporating patient age or weight into dose

331 selection (Aguirre Castaneda et al., 2012; McNally et al., 2015). Despite being well recognized

332 in other areas of pediatric research, the need for age- or weight-based vitamin D dosing has only

333 recently been acknowledged by agencies such as the IOM. Our analysis demonstrated that $90 \%$

334 of trials used a constant dose, with no evidence of a recent shift towards age or weight based 335 practise.

336 Our study findings strongly suggest that identification and synthesis of the clinical trial

337 literature on high dose vitamin D supplementation has been and will continue to be a challenge

338 for clinicians, researchers and policy makers. First, we demonstrated that there is a significant

339 and rapidly expanding body of clinical trial literature. Second, our descriptive analysis identified 
340 significant heterogeneity in multiple relevant study design characteristics, including population

341 (age, disease), dosing regimen, and outcome selection. As many of these characteristics are often

342 poorly described in titles and abstracts, end-users may struggle to not only locate relevant

343 citations but also to determine whether the study will address the question(s). A solution to these

344 two problems was provided as part of this study: a comprehensive online open access searchable

345 database of pediatric clinical trials of high dose vitamin $\mathrm{D}$, similar to what has been generated for

346 other areas, including Eczema (Nankervis, Maplethorpe \& Williams, 2011; Nankervis et al.,

347 2015). Further, we completed a validation study proving the database to have excellent

348 sensitivity, containing 36 of 38 of the pediatric high dose trials reported within 13 published

349 independent systematic reviews. The validation work also identified that the database contained

350 an additional 18 trials that met one or more of the systematic review eligibility criteria. This last

351 finding suggests that researchers performing systematic reviews of vitamin D supplementation

352 may already be struggling with the volume of literature and poor description of trials in titles,

353 abstracts and keywords. In addition to being comprehensive, the database was further designed

354 to assist the end user with heterogeneity. Not only was the database designed to be searchable by

355 key study characteristics, but also the outcome page was set-up to present the user with the study

356 design, population, dosing regimen, and outcome data required to evaluate trial relevance. Utility

357 of the database was also evaluated, by comparing with 4 pediatric systematic reviews, and when

358 combined with the search functions, it was determined that the number of full text articles

359 requiring review could have been reduced by $85 \%$ (Das et al., 2013; Fares et al., 2015; Riverin,

360 Maguire \& Li, 2015; Ali \& McDevitt, 2015). These observations, combined with the fact that the

361 database would reduce the time associated with developing and performing a literature search,

362 indicate the database to be a beneficial resource for the field. 
Finally, the availability of a comprehensive validated database allowed us to identify those

364 areas where there may be sufficient evidence to answer questions regarding the clinical benefits

365 of high-dose vitamin D. Considering all low-risk of bias studies, regardless of size, there were

366 only two areas (respiratory infection/asthma, $n=2166$ and prematurity/low birth weight, $n=2127$ )

367 with more than 100 total children enrolled in the high-dose arms. Compared to reviews of other

368 areas of pediatric research, the average number of children recruited per study was smaller

369 (Hamm et al., 2010). The reason for this is unclear, but given the recent interest in the non-

370 classical roles of vitamin D, much of the identified work may represent pilot work intended to

371 precede large phase III studies (Randolph \& Lacroix, 2002; Nicholson et al., 2003). Of the two

372 areas with a relative breadth of evidence, pediatric respiratory illness and asthma have been

373 recognized by research groups, culminating in multiple systematic reviews (Charan et al., 2012;

374 Das et al., 2013; Pojsupap et al., 2015). Due to heterogeneity in population, dosing regimen and

375 outcome characteristics these reviews were suggestive, but not definitive, for benefit and further

376 research is underway. In contrast to the area of pediatric respiratory illness, there have not been

377 any recent attempts to systematically synthesize the significant clinical trial literature in the area

378 of prematurity and/or low birth weight. A systematic review of the effectiveness and safety in

379 this population may be worthwhile as metabolic bone disease remains a problem and recent

380 observational data suggests that vitamin D deficiency may augment non-musculoskeletal

381 pathophysiology (Onwuneme et al., 2012). This work would benefit nutrition guidelines in

382 NICU and inform dosing regimens for future phase III studies. Finally, it is important to note that

383 with somewhere between one and two dozen new publications per year the areas with sufficient

384 high quality evidence to address clinical efficacy could change quickly. 
Although this review has many strengths, a number of important limitations should be

386

387

388

389

390

391

392

393

394

395

396

397

398

399

400

401

402

403

404 405 406

acknowledged. First, for the majority of the trials information was not available on potentially

relevant study characteristics including race, UV exposure, diet, drug compliance and blood

collection techniques. Second, the large volume of studies and significant heterogeneity in

relevant study characteristics presented considerable challenges for synthesis and presentation of

the studies in a manner useful to all clinicians and researchers. As has been successfully

performed in other clinical areas, including eczema and pediatric intensive care, we sought to

address this problem through the creation of a comprehensive accessible online searchable

database of identified trials (Nankervis, Maplethorpe \& Williams, 2011; Duffett et al., 2013).

Using this database readers and end-users should be able to quickly locate and evaluate the specific population, dosing regimen, outcome and/or study design features of interest. The searchable database also helps address another important study limitation created by the lack of

an accepted definition or algorithm for high dose vitamin D. Recognizing the challenges

involved with developing such a definition, we chose to use a very inclusive or sensitive

threshold. We recognize that the true definition of high dose also incorporates dosing and patient factors including age, disease status, and dose frequency and duration. We have carefully designed the database to ensure that users have the ability to rapidly evaluate these characteristics and apply their own thresholds or algorithm for defining high dose. Finally, a significant limitation of the database is that it is at risk for going out of date. Our goal is to update this list yearly, and provide a data entry page for research groups to provide relevant information on their study.

\section{Conclusion}


vitamin D, and made relevant trial information available as part of an online searchable database.

Importantly, this field has seen an increase in published trials over the past decade, spanning a

410 wide range of populations, dosing regimens and outcomes. To assist the field we developed,

411 validated and demonstrated utility of an online database of pediatric clinical trials of high dose

412 supplementation vitamin D development. The availability of this database, combined with search

413 functions and extracted data, should aid clinicians, researchers and policy makers. Using this

414 resource, clinicians will be able to quickly and comprehensively identify and evaluate the level

415 of clinical trial evidence for a particular patient population. Finally, the availability of an up-to-

416 date list of published trials, combined with extracted information on population, eligibility

417 criteria, dosing regimen and outcomes should expedite the systematic review process for

418 researchers and policy makers.

419

\section{References}

421

422

423

424

425

426

427

428

429

430

431

432

433

434

435

436

437

438

Abrams SA, Coss-Bu JA, Tiosano D 2013. Vitamin D: effects on childhood health and disease. Nature reviews. Endocrinology 9:162-170.

Aguirre Castaneda R, Nader N, Weaver A, Singh R, Kumar S 2012. Response to vitamin D3 supplementation in obese and non-obese Caucasian adolescents. Hormone research in pdiatrics 78:226-231.

Ahmed SF, Franey C, McDevitt H, Somerville L, Butler S, Galloway P, Reynolds L, Shaikh MG, Wallace AM 2011. Recent trends and clinical features of childhood vitamin D deficiency presenting to a children's hospital in Glasgow. Archives of Disease in Childhood 96:694-696.

Ali SR, McDevitt H 2015. Question 1: does vitamin D supplementation prevent acute lower respiratory tract infections in children? Archives of Disease in Childhood 100:892-895.

Bastian H, Glasziou P, Chalmers I 2010. Seventy-five trials and eleven systematic reviews a day: how will we ever keep up? PLoS medicine 7:e1000326.

Beck-Nielsen SS, Jensen TK, Gram J, Brixen K, Brock-Jacobsen B 2009. Nutritional rickets in Denmark: a retrospective review of children's medical records from 1985 to 2005. European Journal of Pediatrics 168:941-949.

Brehm JM, Schuemann B, Fuhlbrigge AL, Hollis BW, Strunk RC, Zeiger RS, Weiss ST, Litonjua AA, Childhood Asthma Management Program Research Group 2010. Serum 
439

440

441

442

443

444

445

446

447

448

449

450

451

452

453

454

455

456

457

458

459

460

461

462

463

464

465

466

467

468

469

470

471

472

473

474

475

476

477

478

479

480

481

482

483

484

vitamin D levels and severe asthma exacerbations in the Childhood Asthma Management Program study. Journal of Allergy \& Clinical Immunology 126:52-8.e5.

Cadario F, Prodam F, Savastio S, Monzani A, Balafrej A, Bellomo G, Bona G 2015. Vitamin D status and Type 1 diabetes in children: evaluation according to latitude and skin colour. Minerva pediatrica 67:263-267.

Canadian Agency for Drugs and Technologies in Health 2015. Vitamin D Testing in the General Population: A Review of the Clinical and Cost-Effectiveness and Guidelines.

Carlsson T, Bergman G, Karlsson A-M, Mattsson E 2015. Content and quality of information websites about congenital heart defects following a prenatal diagnosis. Interactive journal of medical research 4:e4.

Charan J, Goyal JP, Saxena D, Yadav P 2012. Vitamin D for prevention of respiratory tract infections: A systematic review and meta-analysis. Journal of pharmacology \& pharmacotherapeutics 3:300-303.

Das RR, Singh M, Panigrahi I, Naik SS 2013. Vitamin d supplementation for the treatment of acute childhood pneumonia: a systematic review. ISRN pediatrics 2013:459160-7.

Duffett M, Choong K, Hartling L, Menon K, Thabane L, Cook DJ 2013. Randomized controlled trials in pediatric critical care: a scoping review. Critical care (London, England) 17:R256.

Fares MM, Alkhaled LH, Mroueh SM, Akl EA 2015. Vitamin D supplementation in children with asthma: a systematic review and meta-analysis. BMC research notes 8:23.

Godel J 2007. Vitamin D supplementation: Recommendations for Canadian mothers and infants. Paediatrics \& child health 12:583-598.

Gray K, Wood N, Gunasekera H, Sheikh M, Hazelton B, Barzi F, Isaacs D 2012. Vitamin d and tuberculosis status in refugee children. Pediatric Infectious Disease Journal 31:521-523.

Hamm MP, Hartling L, Milne A, Tjosvold L, Vandermeer B, Thomson D, Curtis S, Klassen TP 2010. A descriptive analysis of a representative sample of pediatric randomized controlled trials published in 2007. BMC Pediatrics 10:96.

Harris PA, Taylor R, Thielke R, Payne J, Gonzalez N, Conde JG 2009. Research electronic data capture (REDCap)--a metadata-driven methodology and workflow process for providing translational research informatics support. Journal of biomedical informatics 42:377-381.

Hathcock JN, Shao A, Vieth R, Heaney R 2007. Risk assessment for vitamin D. American Journal of Clinical Nutrition 85:6-18.

Higgins JPT, Green S 2011. Cochrane Handbook for Systematic Reviews of Interventions. John Wiley \& Sons.

Küçükdurmaz F, Gomez MM, Secrist E, Parvizi J 2015. Reliability, Readability and Quality of Online Information about Femoracetabular Impingement. Archives of bone and joint surgery 3:163-168.

Levin AD, Wadhera V, Leach ST, Woodhead HJ, Lemberg DA, Mendoza-Cruz AC, Day AS 2011. Vitamin D deficiency in children with inflammatory bowel disease. Digestive diseases and sciences 56:830-836.

Manaseki-Holland S, Qader G, Isaq Masher M, Bruce J, Zulf Mughal M, Chandramohan D, Walraven G 2010. Effects of vitamin D supplementation to children diagnosed with pneumonia in Kabul: a randomised controlled trial. Tropical medicine \& international health : TM \& IH 15:1148-1155.

Markestad T, Hesse V, Siebenhuner M, Jahreis G, Aksnes L, Plenert W, Aarskog D 1987. Intermittent high-dose vitamin D prophylaxis during infancy: effect on vitamin D metabolites, calcium, and phosphorus. American Journal of Clinical Nutrition 46:652-658. 
485

486

487

488

489

490

491

492

493

494

495

496

497

498

499

500

501

502

503

504

505

506

507

508

509

510

511

512

513

514

515

516

517

518

519

520

521

522

523

524

525

526

527

528

529

530

McNally JD, Iliriani K, Pojsupap S, Sampson M, O'Hearn K, McIntyre L, Fergusson D, Menon K 2015. Rapid Normalization of Vitamin D Levels: A Meta-Analysis. Pediatrics 135:e152e166.

McNally JD, Menon K, Chakraborty P, Fisher L, Williams KA, Al-Dirbashi OY, Doherty DR, Canadian Critical Care Trials Group 2012. The association of vitamin D status with pediatric critical illness. Pediatrics 130:429-436.

Melamed ML, Kumar J 2010. Low levels of 25-hydroxyvitamin D in the pediatric populations: prevalence and clinical outcomes. Pediatric health 4:89-97.

Merewood A, Mehta SD, Grossman X, Chen TC, Mathieu J, Holick MF, Bauchner H 2012. Vitamin D status among 4-month-old infants in New England: a prospective cohort study. Journal of Human Lactation 28:159-166.

Moher D, Liberati A, Tetzlaff J, Altman DG, PRISMA Group 2009. Preferred reporting items for systematic reviews and meta-analyses: the PRISMA statement. PLoS medicine 6:e1000097.

Munns C, Zacharin MR, Rodda CP, Batch JA, Morley R, Cranswick NE, Craig ME, Cutfield WS, Hofman PL, Taylor BJ, Grover SR, Pasco JA, Burgner D, Cowell CT, Paediatric Endocrine Group, Paediatric Bone Australasia 2006. Prevention and treatment of infant and childhood vitamin D deficiency in Australia and New Zealand: a consensus statement. In: 268-272.

Nankervis H, Devine A, Williams HC, Ingram JR, Doney E, Delamere F, Smith S, Thomas KS 2015. Validation of the global resource of eczema trials (GREAT database). BMC dermatology 15:4.

Nankervis H, Maplethorpe A, Williams HC 2011. Mapping randomized controlled trials of treatments for eczema--the GREAT database (the Global Resource of EczemA Trials: a collection of key data on randomized controlled trials of treatments for eczema from 2000 to 2010). BMC dermatology 11:10.

Nicholson CE, Gans BM, Chang AC, Pollack MM, Blackman J, Giroir BP, Wilson D, Zimmerman JJ, Whyte J, Dalton HJ, Carcillo JA, Randolph AG, Kochanek PM 2003. Pediatric critical care medicine: planning for our research future. Pediatric critical care medicine : a journal of the Society of Critical Care Medicine and the World Federation of Pediatric Intensive and Critical Care Societies 4:196-202.

Onwuneme C, Carroll A, McCarthy R, Kilbane M, McKenna M, Murphy N, Molloy EJ 2012. Towards evidence based medicine for paediatricians. Question 2. What is the ideal dose of vitamin D supplementation for term neonates? Archives of Disease in Childhood 97:387389.

Parfitt AM, Gallagher JC, Heaney RP, Johnston CC, Neer R, Whedon GD 1982. Vitamin D and bone health in the elderly. American Journal of Clinical Nutrition 36:1014-1031.

Pithon MM, Santos dos ES 2014. Information available on the internet about pain after orthognathic surgery: a careful review. Dental press journal of orthodontics 19:86-92.

Pojsupap S, Iliriani K, Sampaio TZAL, O'Hearn K, Kovesi T, Menon K, McNally JD 2015. Efficacy of high-dose vitamin $\mathrm{D}$ in pediatric asthma: a systematic review and meta-analysis. The Journal of asthma : official journal of the Association for the Care of Asthma 52:382390.

Provincial Programs Branch, Goverment of Ontario 2010. OHIP-insured Vitamin D Testing. Randolph AG, Lacroix J 2002. Randomized clinical trials in pediatric critical care: Rarely done but desperately needed. Pediatric critical care medicine : a journal of the Society of Critical 
531

532

533

534

535

536

537

538

539

540

541

542

543

544

545

546

547

548

549

550

551

552

553

554

555

556

557
Care Medicine and the World Federation of Pediatric Intensive and Critical Care Societies 3:102-106.

Redmond CE, Nason GJ, Kelly ME, McMahon C, Cantwell CP, Quinlan DM 2015. Transrectal Ultrasound Guided Biopsy of the Prostate: Is the Information Accessible, Usable, Reliable and Readable? Current urology 8:32-37.

Riverin BD, Maguire JL, Li P 2015. Vitamin D Supplementation for Childhood Asthma: A Systematic Review and Meta-Analysis. PLoS ONE [Electronic Resource] 10:e136841.

Robinson PD, Högler W, Craig ME, Verge CF, Walker JL, Piper AC, Woodhead HJ, Cowell CT, Ambler GR 2006. The re-emerging burden of rickets: a decade of experience from Sydney. Archives of Disease in Childhood 91:564-568.

Ross AC, Manson JE, Abrams SA, Aloia JF, Brannon PM, Clinton SK, Durazo-Arvizu RA, Gallagher JC, Gallo RL, Jones G, Kovacs CS, Mayne ST, Rosen CJ, Shapses SA 2011. The 2011 report on dietary reference intakes for calcium and vitamin D from the Institute of Medicine: what clinicians need to know. Journal of Clinical Endocrinology \& Metabolism 96:53-58.

Sampson M, McGowan J, Cogo E, Grimshaw J, Moher D, Lefebvre C 2009. An evidence-based practice guideline for the peer review of electronic search strategies. Journal of clinical epidemiology 62:944-952.

Thacher TD, Fischer PR, Pettifor JM 2014. Vitamin D treatment in calcium-deficiency rickets: a randomised controlled trial. Archives of Disease in Childhood 99:807-811.

Tomaino K, Romero KM, Robinson CL, Baumann LM, Hansel NN, Pollard SL, Gilman RH, Mougey E, Lima JJ, Checkley W, PURA study investigators 2015. Association Between Serum 25-Hydroxy Vitamin D Levels and Blood Pressure Among Adolescents in Two Resource-Limited Settings in Peru. American journal of hypertension 28:1017-1023.

Tsay M-Y, Yang Y-H 2005. Bibliometric analysis of the literature of randomized controlled trials. Journal of the Medical Library Association: JMLA 93:450-458. 
1

Flow chart of study selection based on inclusion and exclusion criteria.

The stages of a systematic selection scheme include: identification, screening, eligibility, and final included studies. 


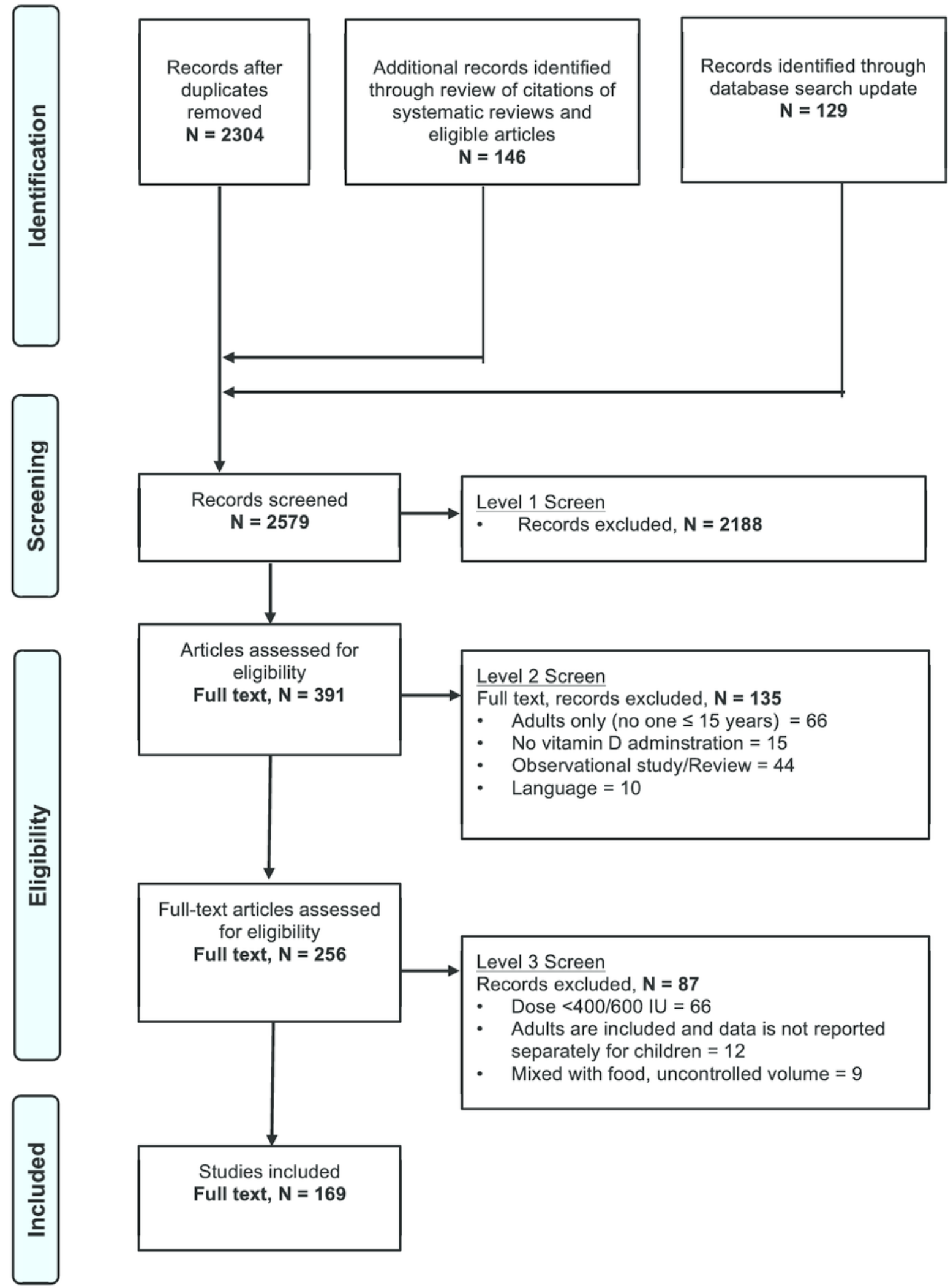


2

Evolution of pediatrics trials of high dose vitamin D over time.

Exponential increase in number of trials $(A, R 2=0.96, p<0.001)$. B) Comparison of studied populations among the different decades $(p<0.001)$. C) Comparison of form of vitamin $D$ administered among different decades $(p<0.001)$. ( $\bullet$ Healthy/subclinical VDD; $(O)$ Classical; ( $\mathbf{v})$ Non-classical; ( $\square$ ) Cholecalciferol; (匹) Ergocalciferol.
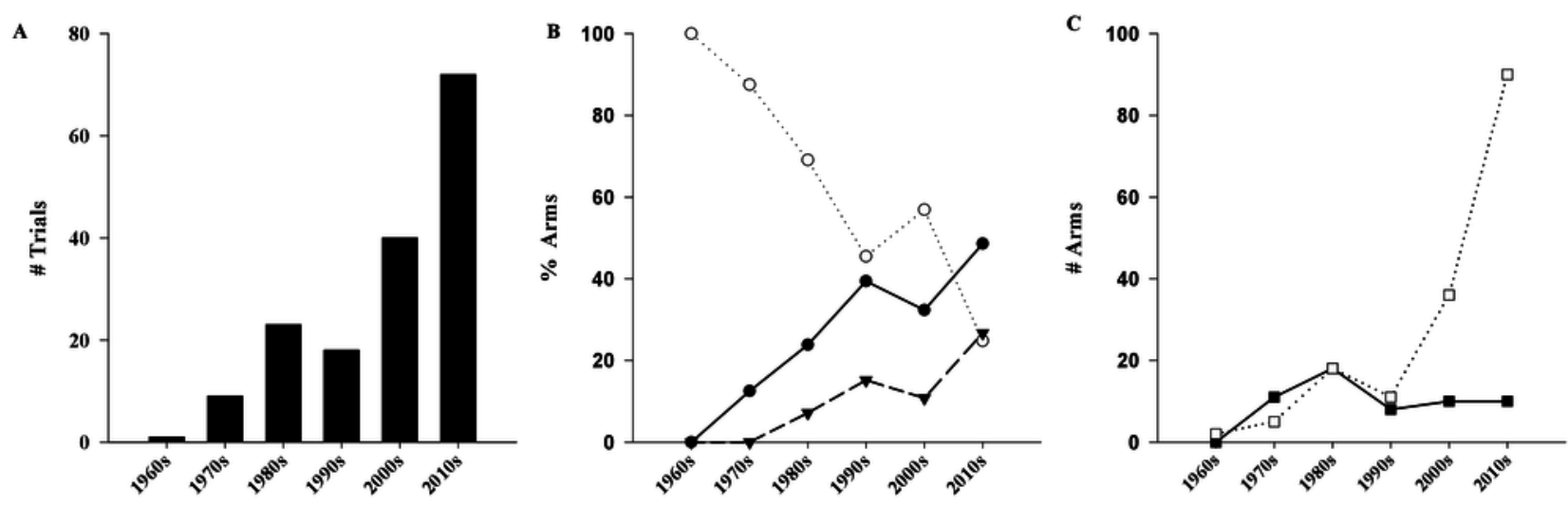


\section{Table $\mathbf{1}$ (on next page)}

Assessment of study design and methodological quality.

Trials enrolled a total of 18539 patients and a median (IQR) of 49 (25 - 94). High dose arms enrolled 11947 patients with a median of 25 (14 - 42). 


\begin{tabular}{lcc}
\hline Study Characteristic & Trials/Populations & $\mathbf{\%}^{\mathbf{a}}$ \\
\hline & & \\
Study design ${ }^{\text {b }}$ & 108 & 66 \\
RCT/qRCT & 42 & 26 \\
Single arm & 13 & 8 \\
Controlled, other & & \\
\hline & & 23 \\
Randomized trial quality ${ }^{\text {b, c }}$ & 38 & 42 \\
Low risk & 69 & 34 \\
Medium risk/unclear & 56 & \\
High risk & & \\
\hline
\end{tabular}

Cochrane risk of bias $b, c, d$

Generation adequate $56 / 57$

$34 / 35$

Concealment adequate

$50 / 62$

$31 / 38$

Blinding adequate

$47 / 25$

$29 / 15$

Outcome report complete

$124 / 36$

$76 / 22$

Outcome not selective

$89 / 6$

$55 / 4$

\begin{tabular}{lrl} 
Age groups e $^{-}$ & & \\
Neonates & 58 & 32 \\
Infants & 35 & 19 \\
Toddlers & 61 & 34 \\
Schoolers & 104 & 57 \\
Adolescents & 82 & 45 \\
\hline
\end{tabular}

2

3 Abbreviations: (Quasi) randomized controlled trial ((q)RCT).

4 a Because of rounding, percentages may not total 100.

5 b Values represent the number of trials, and the percentage out of the 163 identified trials.

6 c Studies were assessed using Cochrane risk of bias tool (Higgins \& Green, 2011)

7 d For the Cochrane assessment, the number of trials where the risk of bias was unclear, we

8 indicated their numbers after the ' $\%$.

9 e Numbers of populations in each age group out of 181 populations. Numbers will add up to more

10 than 181 populations as some included children from two or more age groups. 


\section{Table 2 (on next page)}

Diagnostic categories and outcomes of studied populations.

263 arms were identified as high dose. Populations in these arms were classified as conditions classically or non-classically associated with vitamin D deficiency. Details provided in supplement information. Last column identifies the number of arms that administered high-dose vitamin $\mathrm{D}$ to 50 patients or more, and that were determined to be at a low-risk of bias. 
1

\begin{tabular}{lccc}
\hline Diagnostic Category & Arms & Patients & $\begin{array}{c}\text { \# Trials of Low-Risk of bias } \\
\text { recruiting } \geq \mathbf{5 0} \text { Patients }\end{array}$ \\
\hline Healthy/subclinical VDD & $\mathbf{9 7}$ & $\mathbf{4 6 0 8}$ & $\mathbf{1 1}$ \\
Classical diseases & & & \\
Premature/low birth weight & $\mathbf{1 2 3}$ & $\mathbf{4 1 3 4}$ & $\mathbf{1}$ \\
Rickets & 43 & 2127 & 1 \\
Malabsorption & 15 & 1359 & 0 \\
Epilepsy/seizure & 7 & 319 & 0 \\
Renal disease & 4 & 125 & 0 \\
Other & 6 & 96 & 0 \\
Non-classical diseases & $\mathbf{N o n - C o n v e n t i o n a l ~ O u t c o m e s ~}$ & 0 \\
Obesity & $\mathbf{4 3}$ & $\mathbf{3 2 0 5}$ & $\mathbf{7}$ \\
Asthma & 7 & 213 & 0 \\
Pneumonia / URTI & 4 & 101 & 1 \\
Recurrent acute otitis media & 3 & 2065 & 4 \\
HIV & 3 & 251 & 1 \\
Dental fluorosis & 3 & 65 & 0 \\
Other & 19 & 55 & 0 \\
\hline
\end{tabular}

2

3 Abbreviations: Upper respiratory tract infections (URTI), vitamin D deficiency (VDD).

4 a Tuberculosis. 


\section{Table 3(on next page)}

Characteristics of vitamin D supplementation in the 263 high dose study arms.

Vitamin $D$ dosing regimens were placed into one of three frequency groups (daily, weekly/biweekly, and single/intermittent). Variable dosing regimens administered doses that are dependent on weight, age or body surface area (BSA). 
1

\begin{tabular}{lrc}
\hline Supplementation & Arms & \% $^{\mathbf{a}}$ \\
\hline & & \\
Dosing regimen & 224 & 85.2 \\
Constant & 39 & 14.8 \\
Variable & & \\
& & \\
Dosing groups & 50 & 19.0 \\
RDA/AI to 999 & 73 & 27.8 \\
1000 to 3999 & 33 & 12.6 \\
4000 to 39999 & 107 & 40.7 \\
$\geq 40000$ & & \\
& & \\
Route b & 238 & 90.5 \\
PO & 24 & 9.1 \\
IM/IV & & \\
& & \\
Form c & 162 & 61.6 \\
D3 & 57 & 21.7 \\
D2 & & \\
\hline & & \\
Frequency & 137 & 36.5 \\
Daily & 96 & 11.4 \\
Intermittent/single dose & 30 & \\
Weekly/biweekly & & \\
&
\end{tabular}

2

3 Abbreviations: Adequate intake (AI), body-surface-area (BSA), ergocalciferol (D2),

4 cholecalciferol (D3), enteral dosing (PO), intramuscular (IM), intravenous (IV), recommended 5 daily intake (RDI).

6 a Because of rounding, percentages may not total 100.

7 b In $1 \mathrm{arm}$, the route was unclear.

$8 \quad$ c In 44 cases vitamin D form was nuclear. 


\section{3}

Comparison of trials among geographical regions.

Number of published trials per region $(A, p<0.001)$, and patients $(B, p<0.001)$. $C-F)$ North America and Europe $(\square)$ compared to the other regions $(\square)$, in terms of route $(C, p<0.001)$, form ( $D, p=0.003)$, dosage $(E, p<0.001)$, frequency of supplementation $(F, p<0.001)$, and population ( $G, p=0.81$ ). Al: Adequate Intake; $C / S$ : Central/southern; D2: Ergocalciferol; D3: Cholecalciferol; IM: Intramuscular; IV: Intravenous; NZ: New Zealand; PO: Oral; RDA: Recommended Dietary Allowance.
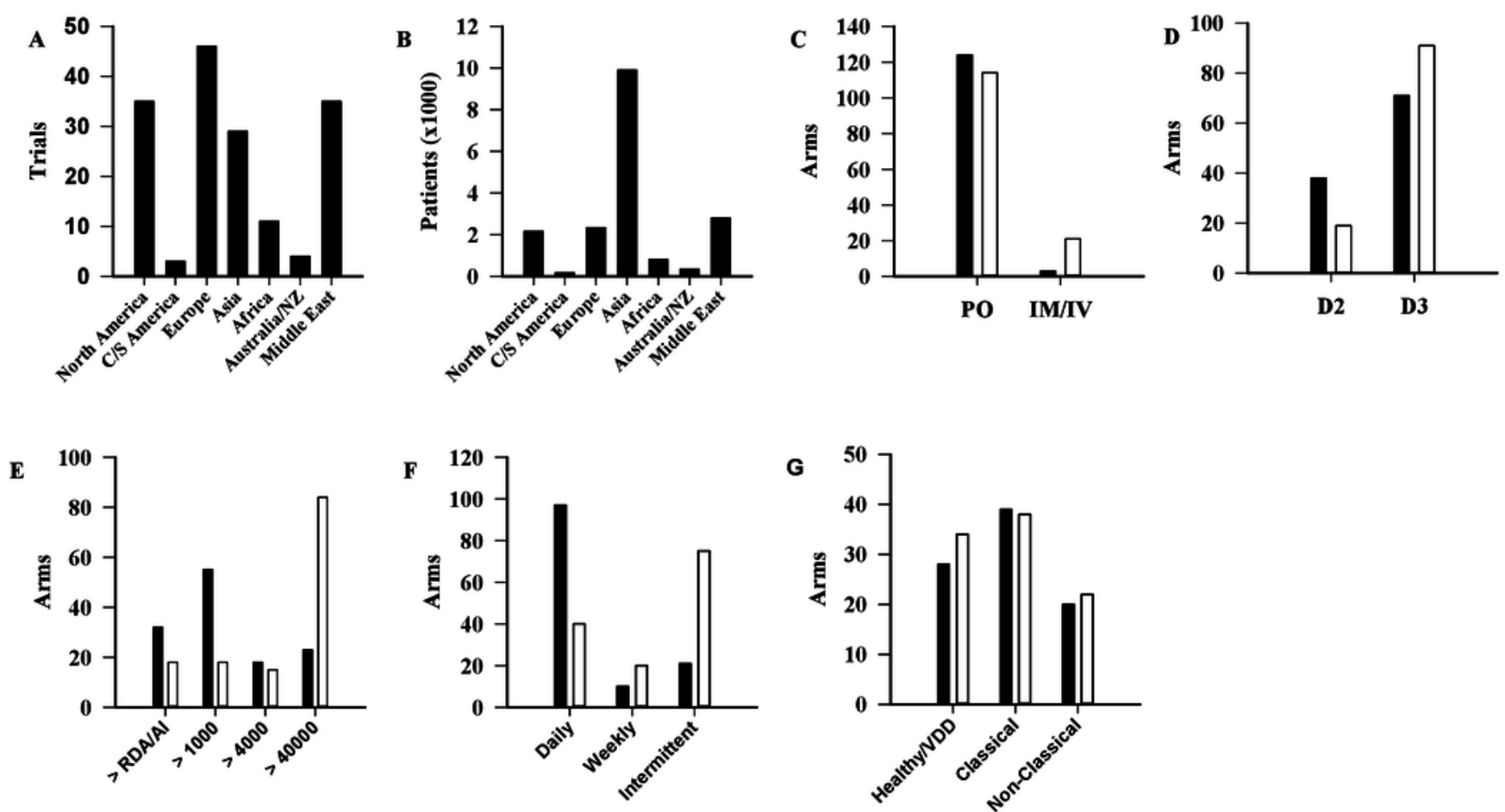
Table 4(on next page)

Classification of studied outcomes of the 163 identified trials. 
1

\begin{tabular}{|c|c|c|}
\hline Outcome & Trials & $\%^{a}$ \\
\hline \multicolumn{3}{|l|}{ Primary Outcome b } \\
\hline$\overline{\text { Biochemical }}$ & 106 & 65 \\
\hline Clinical & 62 & 38 \\
\hline Rickets/bone muscle mass & 35 & 21 \\
\hline Non-classical clinical outcomes & 27 & 17 \\
\hline \multicolumn{3}{|l|}{ Total Outcomes } \\
\hline$\overline{\text { Biochemical }}$ & 156 & 96 \\
\hline 25OHD & 133 & 82 \\
\hline Blood calcium & 118 & 72 \\
\hline Phosphate & 80 & 49 \\
\hline PTH & 69 & 42 \\
\hline ALP & 65 & 40 \\
\hline Urine calcium & 41 & 25 \\
\hline $1,25-(\mathrm{OH}) 2-\mathrm{D}$ & 30 & 18 \\
\hline \multirow[t]{2}{*}{ Calcium absorption } & 7 & 4 \\
\hline & 99 & 61 \\
\hline \multicolumn{3}{|l|}{ Clinical } \\
\hline Bone mass & 47 & 29 \\
\hline Rickets & 33 & 20 \\
\hline Immuno-inflammatory & 19 & 12 \\
\hline Respiratory & 9 & 6 \\
\hline Cardiovascular & 8 & 5 \\
\hline Renal & 6 & 4 \\
\hline Diabetic & 6 & 4 \\
\hline Hematological & 4 & 2 \\
\hline \multicolumn{3}{|l|}{ Other } \\
\hline Anthropological measures & 20 & 12 \\
\hline Adverse effects & 13 & 8 \\
\hline
\end{tabular}

2

3 Abbreviations: Alkaline phosphates (ALP), parathyroid hormone (PTH).

4 a Because of rounding, percentages may not total 100.

5 b Primary outcomes count exceeds 163, as 5 trials had both clinical and biochemical primary

6 outcomes.

7

8 


\section{Table 5 (on next page)}

Validation of the online database using 13 systematic reviews.

Comprehensiveness of the database was evaluated using the search results from 13 systematic reviews not included in the original literature search (2008-2015). 


\begin{tabular}{|c|c|c|c|c|c|c|}
\hline Review & $\begin{array}{l}\text { Trials in the } \\
\text { review }^{\mathrm{a}}\end{array}$ & $\begin{array}{l}\text { Eligible } \\
\text { Trials }^{b}\end{array}$ & $\begin{array}{l}\text { Trials on } \\
\text { ODB }^{c}\end{array}$ & RCTs missing & Sensitivity & Additional trials on ODB \\
\hline 1. Bacchetta, J 2008 & 3 & 0 & 0 & 3: Dose $\leq \mathrm{RDA}$ & 0/0 (NA) & 0 \\
\hline 2. Das, JK 2013 & 5 & 1 & 2 & 4: Dose $\leq \mathrm{RDA}$ & $1 / 1(100 \%)$ & 1 (Vervel 1997) \\
\hline 3. Das, RR 2013 & 2 & 2 & 2 & 0 & $2 / 2(100 \%)$ & 0 \\
\hline 4. Fares, MM 2015 & 4 & 2 & 2 & 2: Dose $\leq \mathrm{RDA}$ & $2 / 2(100 \%)$ & 0 \\
\hline 5. Zittermann, A 2014 & 13 & 10 & 15 & 3: Dose $\leq \mathrm{RDA}$ & $10 / 10(100 \%)$ & $\begin{array}{l}5 \text { (Guillemant 1998, El-Hajj 2006, Dahifar } \\
\text { 2007, Arabi 2009, Majak 2009) }\end{array}$ \\
\hline 6. Riverin, BD 2015 & 8 & 6 & 5 & $\begin{array}{l}1 \text { Reference NA (Darabi 2013) } \\
\text { 2: Dose } \leq \text { RDA }\end{array}$ & $5 / 6(83.3 \%)$ & 0 \\
\hline 7. Ali, SR 2015 & 5 & 3 & 3 & $2:$ Dose $\leq$ RDA & $3 / 3(100 \%)$ & 0 \\
\hline 8. Kerley, CP 2015 & 7 & 6 & 5 & $\begin{array}{l}\text { 1: Dose } \leq \text { RDA } \\
1 \text { Full text NA (Utz 1976) }\end{array}$ & $5 / 6(83.3 \%)$ & 0 \\
\hline 9. Hoffmann, MR 2015 & 1 & 1 & 1 & 0 & $1 / 1(100 \%)$ & 0 \\
\hline $\begin{array}{l}\text { 10. Jamka, M } 2015 \text { (Sci } \\
\text { Rep.) }\end{array}$ & 1 & 1 & 5 & 0 & $1 / 1(100 \%)$ & $\begin{array}{l}4 \text { (Ashraf 2011, Kelishadi 2014, Poomthavorn } \\
\text { 2014, Nader 2014) }\end{array}$ \\
\hline $\begin{array}{l}\text { 11. Jamka, M } 2015 \text { (Eur } \\
\text { J Nutr.) }\end{array}$ & 2 & 2 & 3 & 0 & $2 / 2(100 \%)$ & 1 (Belenchia 2013) \\
\hline 12. Zittermann, A 2015 & 4 & 3 & 7 & 1: Dose $\leq \mathrm{RDA}$ & $3 / 3(100 \%)$ & $\begin{array}{l}4 \text { (Morcos 1998, El-Hajj 2006, Arabi 2009, } \\
\text { Lewis 2015) }\end{array}$ \\
\hline 13. Jiang, W 2015 & 1 & 1 & 1 & 0 & $1 / 1(100 \%)$ & 0 \\
\hline Total & 56 & 38 & 51 & $\begin{array}{l}\text { 18: Dose } \leq \text { RDA } \\
1 \text { Full text NA } \\
1 \text { Reference NA }\end{array}$ & $36 / 38(94.7 \%)$ & 15 \\
\hline
\end{tabular}

1

2 Abbreviations: ODB: Online database (https://vitamind.knackhq.com/pediatrics).

3 a Counting only perspective trials that fell under the pediatrics range and that administered vitamin D.

$4{ }^{\mathrm{b}}$ Trials that satisfied our inclusion criteria of being controlled prospective trial, administering a high dose of vitamin $\mathrm{D}$ to children.

$5 \quad{ }^{\mathrm{c}}$ Counting only those published prior to the search dates of the systematic reviews. 